\title{
Temple syndrome: comprehensive molecular and clinical findings in 32 Japanese patients
}

\author{
Masayo Kagami, MD, PhD ${ }^{1}$, Keisuke Nagasaki, MD, PhD², Rika Kosaki, MD, $\mathrm{PhD}^{3}$, \\ Reiko Horikawa, MD, $\mathrm{PhD}^{4}$, Yasuhiro Naiki, MD, $\mathrm{PhD}^{4}$, Shinji Saitoh, MD, $\mathrm{PhD}^{5}$, \\ Toshihiro Tajima, MD, PhD ${ }^{6}$, Tohru Yorifuji, MD, PhD ${ }^{7}$, Chikahiko Numakura, MD, PhD ${ }^{8}$, \\ Seiji Mizuno, MD, $\mathrm{PhD}^{9}$, Akie Nakamura, MD, $\mathrm{PhD}^{1}$, Keiko Matsubara, MD, $\mathrm{PhD}^{1}$, \\ Maki Fukami, MD, $\mathrm{PhD}^{1}$ and Tsutomu Ogata, MD, $\mathrm{PhD}^{1,10}$
}

\begin{abstract}
Purpose: Temple syndrome (TS14) is a rare imprinting disorder caused by aberrations at the $14 \mathrm{q} 32.2$ imprinted region. Here, we report comprehensive molecular and clinical findings in 32 Japanese patients with TS14.

Methods: We performed molecular studies for TS14 in 356 patients with variable phenotypes, and clinical studies in all TS14 patients, including 13 previously reported.

Results: We identified 19 new patients with TS14, and the total of 32 patients was made up of 23 patients with maternal uniparental disomy (UPD(14)mat), six patients with epimutations, and three patients with microdeletions. Clinical studies revealed both Prader-Willi syndrome (PWS)-like marked hypotonia and Silver-Russell syndrome (SRS)-like phenotype in $50 \%$ of patients, PWS-like hypotonia alone in $20 \%$ of patients, SRS-like phenotype
\end{abstract}

alone in $20 \%$ of patients, and nonsyndromic growth failure in the remaining $10 \%$ of patients in infancy, and gonadotropindependent precocious puberty in $76 \%$ of patients who were pubescent or older.

Conclusion: These results suggest that TS14 is not only a genetically diagnosed entity but also a clinically recognizable disorder. Genetic testing for TS14 should be considered in patients with growth failure plus both PWS-like hypotonia and SRS-like phenotypes in infancy, and/or precocious puberty, as well as a familial history of KagamiOgata syndrome due to maternal microdeletion at $14 \mathrm{q} 32.2$.

Genet Med advance online publication 22 June 2017

Key Words: clinical diagnosis; epimutation; microdeletion; Temple syndrome; UPD(14)mat

\section{INTRODUCTION}

The human chromosome $14 \mathrm{q} 32.2$ region carries paternally and maternally expressed genes ( $P E G s$ and $M E G s$ ), together with the germ-line-derived DLK1-MEG3 intergenic differentially methylated region (MEG3/DLK1:IG-DMR) and the postfertilization-derived MEG3:TSS-DMR, which function as imprinting control centers in the placenta and the body, respectively (Supplementary Figure S1A online) $)^{1,2}$ (the nomenclature of DMRs is based on recent recommendations). ${ }^{3}$ Consistent with this, maternal uniparental disomy 14 (UPD(14)mat) results in a constellation of clinical features such as growth failure, muscular hypotonia, precocious puberty, feeding difficulties, and small hands and feet. ${ }^{4}$ Similar phenotypes are also caused by epimutations (hypomethylations) and microdeletions affecting the paternally derived imprinted region. ${ }^{4}$

Recently, the term "Temple syndrome" (TS14) (OMIM 616222) has been approved for UPD(14)mat and related conditions. ${ }^{4}$ The diagnosis of TS14 is based primarily on genetic rather than clinical findings. To our knowledge, TS14 has been reported in 65 patients to date (51 patients reviewed by Ioannides et al. $^{4}$ and 14 patients reported thereafter (Supplementary Table S4). Notably, most patients were initially suspected to have Prader-Willi syndrome (PWS) or Silver-Russell syndrome (SRS), ${ }^{4-6}$ consistent with phenotypic overlaps between TS14 and PWS (e.g., growth failure, muscular hypotonia, and small hands and feet) and between TS14 and SRS (e.g., growth failure and feeding difficulties), especially in infancy. Furthermore, although rare, TS14 has also been detected in apparently nonsyndromic patients who were born small for gestational age and manifested persistent short stature (SGA-SS) (birth length and/or weight $\leq-2$ standard deviation score (SDS) and postnatal height $\leq-2$ SDS $)^{7}$

Here, we report comprehensive molecular and clinical findings in 32 Japanese patients with TS14, and discuss several

\footnotetext{
${ }^{1}$ Department of Molecular Endocrinology, National Research Institute for Child Health and Development, Tokyo, Japan; ${ }^{2}$ Department of Homeostatic Regulation and Development, Niigata University Graduate School of Medical and Dental Sciences, Niigata, Japan; ${ }^{3}$ Division of Medical Genetics, National Center for Child Health and Development, Tokyo, Japan; ${ }^{4}$ Division of Endocrinology and Metabolism, National Center for Child Health and Development, Tokyo, Japan; ${ }^{5}$ Department of Pediatrics and Neonatology, Nagoya City University Graduate School of Medical Sciences, Nagoya, Japan; ${ }^{6}$ Department of Pediatrics, Jichi Children's Medical Center, Shimotsuke, Japan; ${ }^{7}$ Division of Pediatric Endocrinology and Metabolism, Children's Medical Center, Osaka City General Hospital, Osaka, Japan; ${ }^{8}$ Department of Pediatrics, Yamagata University School of Medicine, Yamagata, Japan; ${ }^{9}$ Department of Pediatrics, Central Hospital, Aichi Human Service Center, Aichi, Japan; ${ }^{10}$ Department of Pediatrics, Hamamatsu University School of Medicine, Hamamatsu, Japan. Correspondence: Masayo Kagami or Tsutomu Ogata (kagami-ms@ncchd.go.jp) or (tomogata@hama-med.ac.jp)
} 
issues including relative frequency of underlying causes, characteristic clinical findings, and clinical indicators for the genetic testing of TS14.

\section{Ethical approval}

MATERIALS AND METHODS

This study was approved by the Institute Review Board Committee at the National Center for Child Health and Development, and was performed after written informed consent was obtained. We also obtained written informed consent to publish facial photographs of nine patients.

\section{Patients}

This study population consisted of two groups of Japanese patients. One contained a total of 346 hitherto unreported patients referred to us for genetic diagnosis: (i) 102 patients with PWS-like phenotypes in the absence of hypermethylated SNURF:TSS-DMR (previously reported as the SNRPNDMR), ${ }^{8}$ (ii) 115 patients with SRS-like phenotypes in the absence of epimutated (hypomethylated) H19/IGF2:IG-DMR and UPD(7)mat, (iii) 126 patients with apparently SGA-SS phenotypes, and (iv) three patients with TS14-like phenotypes (growth failure and precocious puberty). At the time of referral, these clinical diagnoses were made subjectively by the attending physicians, without comprehensive clinical studies.

The second group consisted of 13 previously reported patients with TS14 (Supplementary Table S1): ${ }^{2,6-10} 6$ patients with UPD(14)mat, 4 with epimutations, and 3 with microdeletions. Of the 10 patients with UPD(14)mat or epimutations, 5 exhibited PWS-like phenotypes, 4 manifested SRS-like phenotypes, and 1 showed the SGA-SS phenotype. The 3 patients with microdeletions were ascertained as such through familial studies of children with Kagami-Ogata syndrome (KOS14). The karyotype was normal in 11 of 12 patients examined. The remaining patient with UPD(14)mat (patient 12) had a 46,XX[18]/47,XX,+mar[8] karyotype. Since the marker chromosome was found to be of paternal $14 \mathrm{q} 11.2-\mathrm{q} 12$ origin, this indicated that the marker chromosome was produced during mitosis independently of the generation of UPD(14)mat. ${ }^{10}$ Patient 23 with 46,XX had mosaic UPD(14)mat (46,XX/46,XX,UPD(14)mat). ${ }^{7}$

\section{Genetic studies}

Molecular studies for the genetic diagnosis of TS14 were performed using leukocyte genomic DNA samples from the 346 patients. The methods employed in this study have been reported previously, as have the probes and primers. ${ }^{2,8,11,12}$ In brief, we sequentially performed: (i) methylation analysis for the MEG3/DLK1:IG-DMR and the MEG3:TSS-DMR by pyrosequencing, (ii) microsatellite analysis for nine loci widely dispersed on chromosome 14, and (iii) deletion analysis by array comparative genomic hybridization using 12,600 probes for the chromosome 14q32.2 imprinted region (Agilent Technologies, Santa Clara, CA) and by fluorescence in situ hybridization using probes for the MEG3/DLK1:IG-DMR and the MEG3:TSS-DMR (Supplementary Figure S1B).
We also carried out (i) single-nucleotide polymorphism (SNP) array analysis using the SurePrint G3 ISCA CGH+SNP Microarray Kit (Agilent Technologies) in patients with apparently full maternal isodisomy for chromosome 14, to examine the presence or absence of cryptic heterodisomic regions; (ii) pyrosequencing analysis for the PWS-related SNURF:TSS-DMR, the SRS-related H19/IGF2:IG-DMR, MEST: alt-TSS-DMR, and PEG10:TSS-DMR, and other imprinting disease-related PLAGL1:alt-TSS-DMR, KCNQ1OT1:TSS-DMR, and $G N A S-A / B$ :TSS-DMR in patients with epimutations, to examine the presence or absence of multilocus imprinting disturbances (MLIDs) as possible underlying factor(s) for phenotypic diversity; and (iii) cytogenetic analysis, to examine the presence or absence of chromosomal aberrations including Robertsonian translocation (these analyses had already been performed in the 13 previously reported patients with TS14).

\section{Clinical studies}

We collected detailed clinical findings for all TS14 patients from attending physicians using a comprehensive questionnaire (Table 1 and Supplementary Table S1). We also collected longitudinal data on growth, bone age (BA), Tanner pubertal stage, endocrine findings, therapeutic interventions, and development. Placental weight was assessed using the gestational age-matched Japanese reference data. ${ }^{13}$ Length/height, weight, occipitofrontal circumference (OFC), and body mass index (BMI) were evaluated using the sex- and age-matched Japanese reference data (http://jspe.umin.jp/medical/keisan.html). Target height (a child's adult height predicted from parental heights) and target range (95\% confidence interval of target height) were calculated from the equations by Ogata et al. ${ }^{14}$ BA was determined by the TW-2 method standardized for Japanese. ${ }^{15}$ Pubertal and endocrine data were compared with those of normal Japanese children. ${ }^{16,17}$ Developmental quotient/intellectual quotient were obtained by the method described in the fourth edition of the Diagnostic and Statistical Manual of Mental Disorders. In addition, we asked the physicians to report any clinical findings not covered by the questionnaire.

We also evaluated the presence or absence of (i) clinical features prompting genetic testing for PWS, ${ }^{18}$ i.e., unexplained hypotonia with poor suck ( $<2$ years), hypotonia with history of poor suck associated with global developmental delay (2-6 years), hypotonia or history of hypotonia with poor suck, global developmental delay, and excessive eating with central obesity (6-12 years), and cognitive impairment, excessive eating with central obesity, and hypogonadotropic hypogonadism and/or typical behavior problems ( $\geq 13$ years) (patients with such age-dependent features were regarded as having PWS-like phenotypes); (ii) Netchine-Harbison scoring system features for SRS, ${ }^{19}$ i.e., SGA, postnatal growth failure, relative macrocephaly at birth, protruding forehead, body asymmetry, and feeding difficulties and/or low body mass index (clinical diagnosis of SRS is made when at least four of the six scoring system features are present; ${ }^{19}$ when not all six scoring system features were available, the diagnosis of SRS was made if at least three of five features were present); and 
Table 1 Clinical manifestations in 32 patients with Temple syndrome

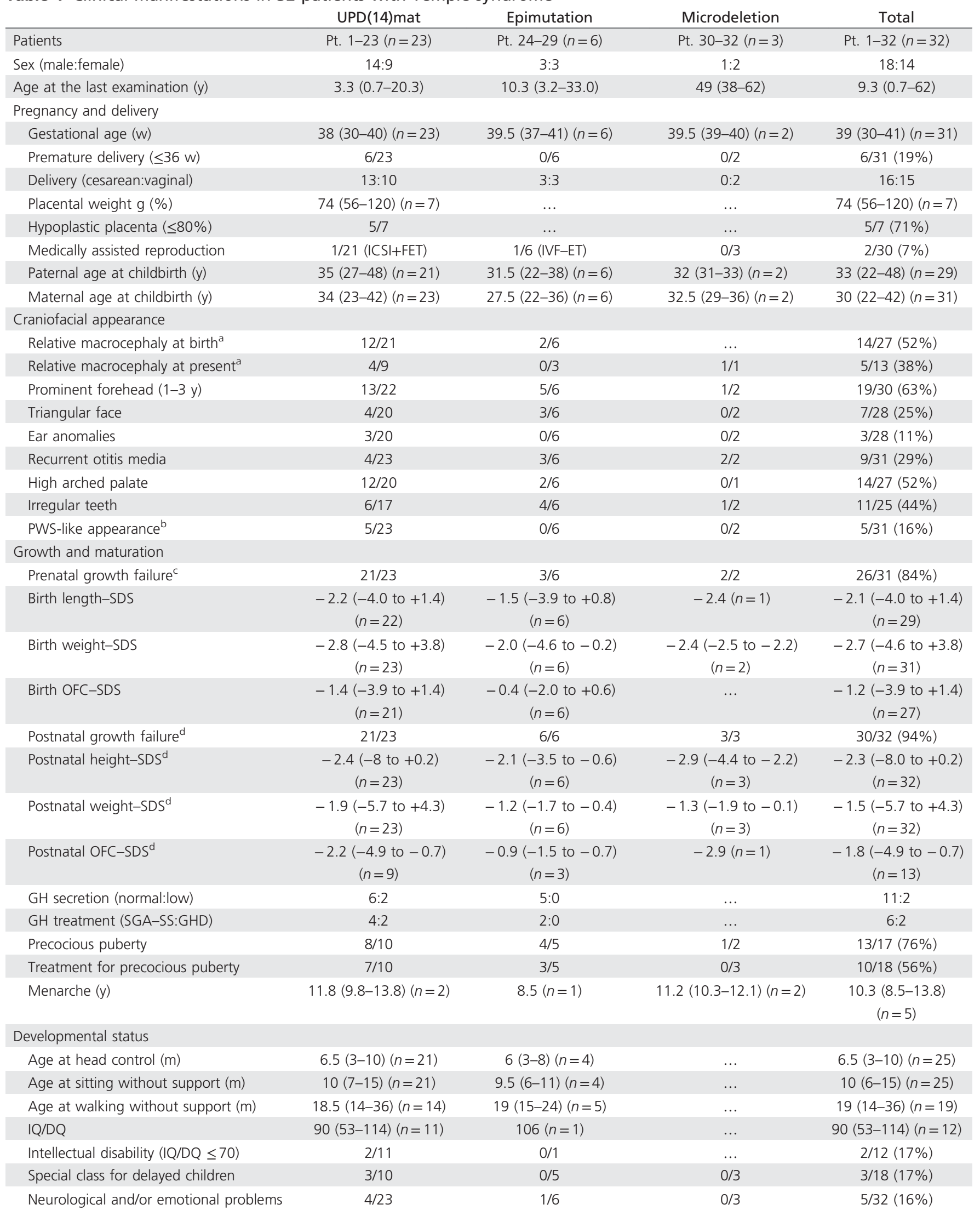




\begin{tabular}{|c|c|c|c|c|}
\hline & UPD(14)mat & Epimutation & Microdeletion & Total \\
\hline \multicolumn{5}{|l|}{ Other findings } \\
\hline Hypotonia (with poor suck) & $16 / 23$ & $4 / 6$ & $1 / 2$ & $21 / 31(68 \%)$ \\
\hline Small hands and/or feet & $20 / 23$ & $6 / 6$ & $3 / 3$ & 29/32 (91\%) \\
\hline Clinodactyly & $8 / 20$ & $3 / 6$ & $0 / 2$ & $11 / 28(39 \%)$ \\
\hline Simian crease & $4 / 18$ & $3 / 6$ & $0 / 2$ & $7 / 26(27 \%)$ \\
\hline Joint hypermobility & $7 / 23$ & $3 / 5$ & $0 / 2$ & $10 / 30(33 \%)$ \\
\hline Feeding difficulties and/or low BMI & $15 / 22$ & $3 / 6$ & $1 / 2$ & $19 / 30(63 \%)$ \\
\hline Undermasculinized genitalia & 5/14 (MP2, CO2, HS1) & $0 / 3$ & $\ldots$ & $5 / 17(29 \%)$ \\
\hline Hypercholesterolemia & $2 / 18$ & $3 / 6$ & $1 / 2$ & $6 / 26(23 \%)$ \\
\hline Diabetes mellitus (type 2) & $1 / 19$ & $1 / 6$ & $1 / 2$ & $3 / 27(11 \%)$ \\
\hline \multicolumn{5}{|l|}{ Reassessed clinical diagnosis in infancy ${ }^{\mathrm{e}}$} \\
\hline SGA-SS phenotype & $3 / 22$ & $0 / 6$ & $0 / 2$ & $3 / 30(10 \%)$ \\
\hline \multicolumn{5}{|l|}{ Reassessed clinical diagnosis from puberty ${ }^{\dagger}$} \\
\hline TS14-like phenotype & $8 / 10$ & $4 / 5$ & $1 / 2$ & $13 / 17(76 \%)$ \\
\hline \multicolumn{5}{|l|}{ PWS: salient features prompting genetic testing } \\
\hline Hypotonia or its history (with poor suck) & $16 / 23$ & $4 / 6$ & $1 / 2$ & $21 / 31(68 \%)$ \\
\hline Global developmental delay ( $\geq 2$ y) & $5 / 17$ & $0 / 6$ & $0 / 3$ & $5 / 26(19 \%)$ \\
\hline Excessive eating with central obesity ( $\geq 6 \mathrm{y})$ & $1 / 10$ & $1 / 5$ & $0 / 3$ & $2 / 18(11 \%)$ \\
\hline Cognitive impairment ( $\geq 13$ y) & $0 / 4$ & $0 / 1$ & $0 / 3$ & $0 / 8(0 \%)$ \\
\hline Hypothalamic hypogonadism ( $\geq 13$ y) & $0 / 4$ & $0 / 1$ & $0 / 3$ & $0 / 8(0 \%)$ \\
\hline Behavior problems ( $\geq 13 \mathrm{y})$ & $0 / 4$ & $0 / 1$ & $0 / 3$ & $0 / 8(0 \%)$ \\
\hline Feeding difficulties and/or low BMI & $15 / 22$ & $3 / 6$ & $1 / 2$ & $19 / 30(63 \%)$ \\
\hline \multicolumn{5}{|l|}{ TS14: salient features } \\
\hline Pre- and/or postnatal growth failure & $22 / 23$ & $6 / 6$ & $3 / 3$ & $31 / 32(97 \%)$ \\
\hline Precocious puberty & $8 / 10$ & $4 / 5$ & $1 / 2$ & $13 / 17(76 \%)$ \\
\hline Treatment for precocious puberty & $7 / 10$ & $3 / 5$ & $0 / 3$ & $10 / 18(56 \%)$ \\
\hline
\end{tabular}

BMI, body mass index; CO, cryptorchidism; ET, embryo transfer; FET, frozen embryo transfer; GH, growth hormone; GHD, growth hormone deficiency; HS, hypospadias; ICSI, intracytoplasmic sperm injection; IQ/DQ, intelligence/developmental quotient; IVF-ET, in vitro fertilization and embryo transfer; MP, micropenis; m, month; OFC, occipitofrontal circumference; PWS, Prader-Willi syndrome; SDS, standard deviation score; SGA-SS, small for gestational age-short stature; SRS, Silver-Russell syndrome; TS14, Temple syndrome; UPD(14)mat, maternal uniparental disomy syndrome.

For the frequency, the denominators indicate the number of patients examined for the presence or absence of each feature, and the numerators represent the number of patients assessed as positive for that feature. Clinical findings of each case are shown in Supplementary Table S1.

${ }^{a}$ Birth OFC SDS $\geq 1.5$ above birth length or weight SDS; present OFC SDS $\geq 1.5$ above present height or weight SDS. ' and a triangular mouth. "Birth length and/or weight $\leq-2$ SDS for gestational age. ${ }^{d}$ Postnatal height $\leq-2$ SDS for age; the latest height data in childhood before the

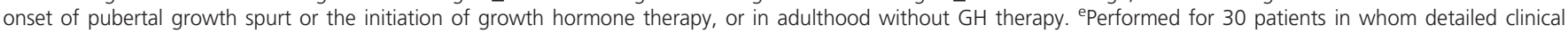
findings for the assessment of both PWS-like features prompting the genetic studies and SRS-like clinical features utilized in the Netchine-Harbison scoring system were

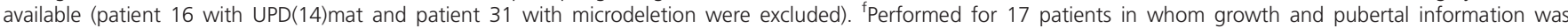
available. ${ }^{9}$ Assessed for patients in whom all the six $\mathrm{N}-\mathrm{H}$ scoring system features have been evaluated.

(iii) clinical features suggestive of TS14, i.e., SGA, postnatal growth failure, and precocious puberty ${ }^{4}$ (patients with all three features were regarded as having TS14-like phenotypes) (Table 1 and Supplementary Table S1).

\section{Statistical analysis}

The statistical significance of the median and frequency of data obtained from patients with UPD(14)mat, those with epimutations, and those with microdeletions were examined 
using the Kruskal-Wallis test and Fisher's exact probability test, respectively. $P<0.05$ was considered significant.

\section{RESULTS}

\section{Genetic studies}

The results are shown in Supplementary Figure S1B. Methylation analysis of the 346 patients revealed hypomethylations of both the MEG3/DLK1:IG-DMR and the MEG3:TSS-DMR in 19 patients. Microsatellite analysis with parental DNA samples showed UPD(14)mat in 17 patients, consisting of 12 patients with trisomy rescue (TR)- or gamete complementation (GC)mediated UPD(14)mat revealed by heterodisomy for at least one locus, 3 patients with monosomy rescue (MR) or postfertilization mitotic error (PE)-mediated UPD(14)mat indicated by full isodisomy, and 2 patients with PE-mediated UPD (14)mat demonstrated by segmental isodisomy or mosaicism for full isodisomy. The remaining 2 patients were assessed as having epimutations because of the lack of a discernible deletion. The 3 patients with MR/PE-mediated UPD(14)mat were confirmed to have full isodisomy by SNP array, and the two patients with epimutations had no definitive MLID in the examined DMRs (Supplementary Table S2). The karyotype was normal in 14 of the 19 patients examined.

Thus, in conjunction with the 13 previously reported patients, ${ }^{2,6-10}$ TS14 was identified in a total of 32 patients: 23 patients with UPD(14)mat (16 with TR/GC type, 4 with $\mathrm{MR} / \mathrm{PE}$ type, and 3 with $\mathrm{PE}$ type), 6 patients with epimutations, and 3 patients with microdeletions (2 with a microdeletion involving $D L K 1$ and 1 with a microdeletion encompassing DLK1 and RTL1) (Table 1, Supplementary Table S1, and Supplementary Figure S1C).

\section{Evaluation of clinical findings}

The data obtained from patients with UPD(14)mat, those with epimutations, and those with microdeletions are summarized in Table 1, and those for each patient are shown in Supplementary Table S1. Phenotypic comparison showed no significant difference among the three groups of patients, except for significantly increased present ages in patients with microdeletions $(P=0.021)$ and significantly advanced maternal childbearing age in patients with UPD(14) mat $(P=0.033)$. In addition, the clinical findings for patient 23 with mosaic UPD(14)mat were grossly similar to those for other patients (Table S1). Thus, we integrated clinical findings for all 32 patients, and performed clinical assessment.

\section{Pregnancy and delivery}

Placental hypoplasia was observed in the deliveries of $~ 70 \%$ of patients, and an exceptionally large placenta was recorded for patient 21. Premature delivery occurred in $\sim 20 \%$ of patients. Two patients (patients 18 and 25) were born after medically assisted reproduction.

\section{Craniofacial appearance}

Facial appearance changed with age (Figure 1). Most patients showed an SRS-like craniofacial appearance with relative macrocephaly and prominent forehead in infancy, but such appearance became obscure with age. High-arched palate and irregular teeth were fairly common, and triangular face, ear anomalies, and recurrent otitis media were rather infrequent. A PWS-like craniofacial appearance, which is not regarded as an indication for genetic studies, ${ }^{18}$ remained uncommon.

\section{Growth and maturation}

Pre- and postnatal growth failure was observed in most patients, although five (patients 14, 22, 24, 27, and 29) lacked prenatal growth failure (birth lengths and weights $>-2$ SDS), and two (patients 11 and 22) were free from postnatal growth failure (present heights $>-2$ SDS at $\sim 2$ years of age, in childhood before the onset of pubertal growth spurt and the initiation of growth hormone (GH) therapy, or in adulthood without GH therapy). The birth weight and present weight of patient 22 were exceptionally large.

Longitudinal growth and maturation data for 15 patients are shown in Figure 2, and endocrine and pubertal findings for 18 patients are summarized in Supplementary Table S3. Most patients showed precocious puberty with rapid BA progression, together with growth failure. $\mathrm{GH}$ provocation tests indicated apparent growth hormone deficiency (GHD) in 2 of 13 patients examined (patients 16 and 23). GH treatment was performed with the dosage for GHD $(0.175 \mathrm{mg} / \mathrm{kg} /$ week $)$ in patients 16 and 23 and with the dosage for SGS-SS $(0.23-0.47 \mathrm{mg} / \mathrm{kg} /$ week) in 7 patients (for details, see the footnote to Supplementary Table S3). GH therapy was associated with accelerated statural growth and progression of BA in most patients, except for patient 23 in whom these phenomena were not observed. Gonadotropinreleasing hormone $(\mathrm{GnRH})$ stimulation tests revealed gonadotropin-dependent central precocious puberty. Thus, GnRH analog therapy was started at various dosages (for details, see the footnote to Supplementary Table S3), suppressing pubertal development. BMIs tended to be low in most patients, especially in infancy, although they were apparently better preserved in TS14 patients than in SRS patients (Supplementary Figure S2). BMIs gradually increased along the reference curves in most patients, although they advanced rather rapidly in several patients (e.g., patients 13 and 27), and patient 22 had an extremely high BMI. In addition, most patients tended to exhibit truncal obesity from late childhood (Figure 1).

\section{Developmental status}

The data for each patient are shown in Supplementary Figure S3. Gross motor development was delayed in most patients, consistent with a high frequency of marked hypotonia. In patients who passed motor developmental milestones, head control was achieved at $\sim 7$ months, sitting without support at $\sim 10$ months, and walking without support at $\sim 19$ months of age. Intellectual disability (ID, defined as a developmental quotient/intelligence quotient $\leq 70)$ and enrollment in special classes were recorded in $17 \%$ of patients. However, of the 4 patients reported to have ID or 


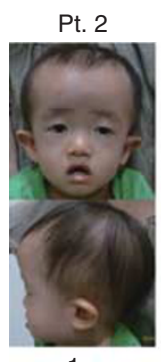

$1 \mathrm{y}$

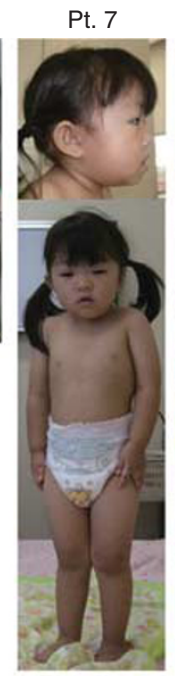

$2 y$
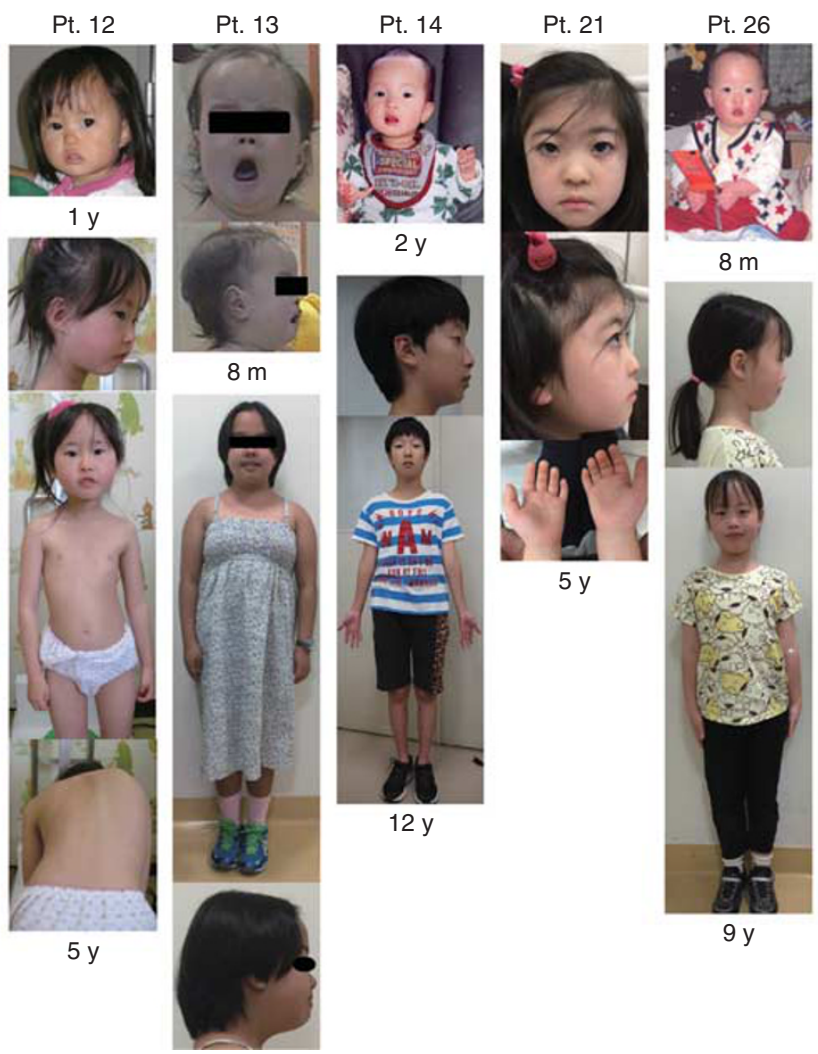

$9 \mathrm{y}$

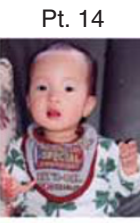

$2 y$
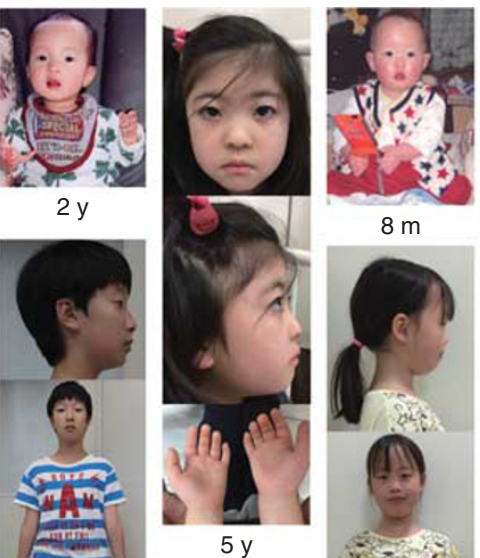

$8 \mathrm{~m}$

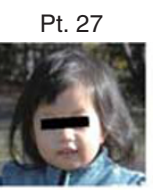

$1 \mathrm{y}$

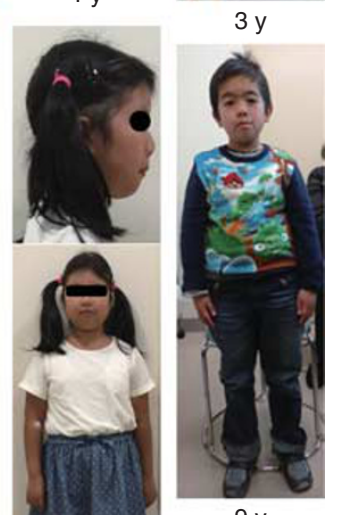

$9 \mathrm{y}$

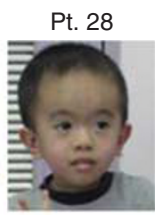

$3 \mathrm{y}$

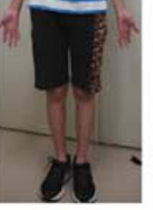

$12 \mathrm{y}$

$12 \mathrm{y}$

Figure 1 Photographs of nine patients with Temple syndrome. Silver-Russell syndrome-like craniofacial appearance became less remarkable with age, and truncal obesity became apparent with age.

enrolled in special classes (patients 10,12,17, and 23), patient 10 had a genetic background for ID (both parents were enrolled in special classes), patient 12 had West syndrome, and patient 23 had a hearing impairment. In addition, 4 patients (patients 16, 22, 23, and 29) were afflicted with neurological and/or emotional problems. While a substantial fraction of patients were suspected to have speech delay in infancy to early childhood, they had apparently normal verbal capacity in childhood (therefore, speech delay was not included in Table 1 and Supplementary Table S1).

\section{Other findings}

Other features included (i) small hands and feet in most patients; (ii) marked hypotonia and feeding difficulties in infancy in approximately two-thirds of patients; (iii) clinodactyly, simian crease, joint hypermobility, body asymmetry, and scoliosis in $\sim 20-40 \%$ of patients; (iv) undermasculinized genitalia in $\sim 30 \%$ of male patients, and (v) hypercholesterolemia in $\sim 20 \%$ of patients and type 2 diabetes mellitus in $\sim 10 \%$ of patients.

\section{Reassessment of clinical diagnosis}

Since the initial clinical diagnoses at the time of referral for genetic studies were made subjectively by the attending physicians, we reassessed clinical diagnoses on the basis of the comprehensive clinical findings (Table 1 and Supplementary Table S1). Detailed clinical findings on the assessment of both the PWS-like features prompting the genetic studies and the SRS-like clinical features utilized in the Netchine-Harbison scoring system were available for 30 patients, the two exceptions being patient 16, in whom a PWS-like phenotype was absent but clinical information for SRS remained fragmentary, and patient 31, in whom infantile clinical information was barely obtained (thus, PWS features and birth length and/or weight were evaluated in 31 patients, including patient 16) (Table 1). Detailed clinical findings for the assessment of TS14-like features, including longitudinal growth and maturation data, were obtained for 17 patients.

Reassessment of clinical features in infancy revealed the presence of both PWS-like and SRS-like phenotypes in 15 patients, a PWS-like phenotype alone in 6 patients, an SRSlike phenotype alone in 6 patients, and the absence of both PWS-like and SRS-like phenotypes (i.e., SGA-SS phenotypes) in the remaining 3 patients (Table 1). The initial diagnosis of the 15 patients with both PWS-like and SRS-like phenotypes was PWS in 9 patients and SRS in 5 patients; the remaining patient was identified through familial study of a child with KOS14 (Supplementary Table S1). This indicated the difficulty of identifying coexisting syndromes. Of PWS-like features prompting genetic diagnosis, marked hypotonia in 

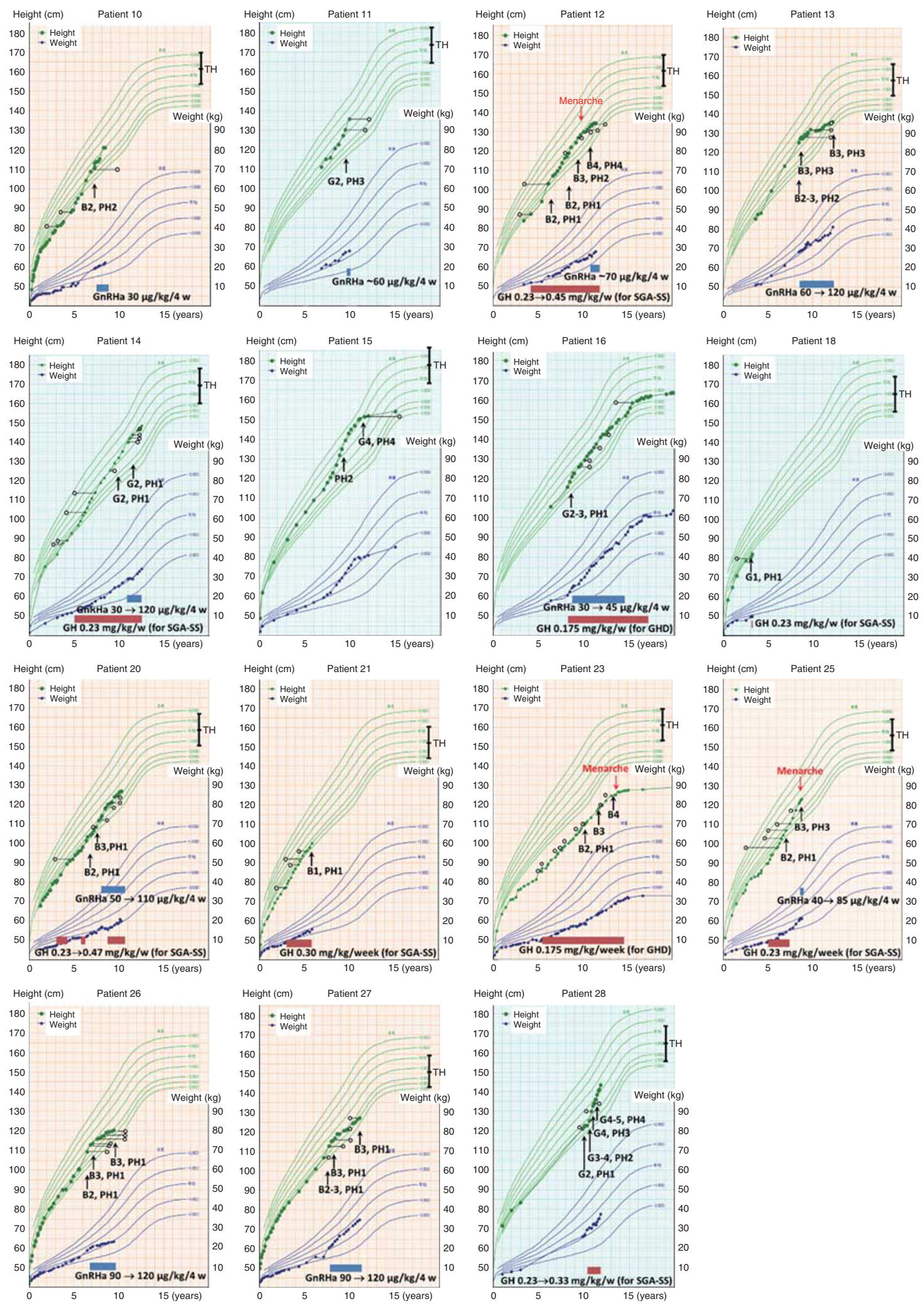

Figure 2 Growth charts for 15 patients with Temple syndrome. B, breast; G, genitalia; GH, growth hormone; GHD, GH deficiency; GnRHa, gonadotropin-releasing hormone analog; PH, pubic hair; SGA-SS, small for gestational age and short in stature; TH, target height. The vertical bars adjacent to $\mathrm{TH}$ indicate target range. 
infancy was prevalent, whereas clinical features characteristic of aged PWS patients ( $\geq 13$ years) were absent. Of the six Netchine-Harbison SRS scoring features, body asymmetry was rather infrequent, whereas the remaining five features, including relative macrocephaly and feeding difficulties, were fairly frequent.

Reassessment of clinical features after pubertal onset showed the presence of pre- and postnatal growth failure and the precocious puberty characteristic of TS14 in 13 of the 17 patients (Table 1). The initial diagnosis for 9 patients referred to us before pubertal onset was PWS in 6 patients, SRS in 2 patients, and SGA-SS in 1 patient, whereas that for 3 patients referred to us after pubertal onset was TS14; the remaining patient was detected through familial study of a child with KOS14 (Supplementary Table S1). This indicated the difficulty of diagnosing TS14 before pubertal onset.

\section{DISCUSSION}

After examining 346 patients, we identified 19 new patients with molecularly confirmed TS14. Since the 346 patients had been screened using positive clinical findings and previous molecular studies on PWS and SRS, the prevalence of TS14 in the general population remains unknown. Nevertheless, this study demonstrates that TS14 is present with a low but certain frequency.

The relative frequency of underlying causes of TS14 was $72 \%$ for UPD(14)mat, $19 \%$ for epimutations, and $9 \%$ for microdeletions. While the results are grossly similar to those of the previous review, ${ }^{4}$ in this study the frequency of UPD (14)mat was relatively low and that of epimutations relatively high. This would be due primarily to the historical fact that UPD(14)mat was once preferentially found by genotyping analysis for patients with Robertsonian translocations, ${ }^{20}$ whereas epimutations as well as UPD(14)mat and microdeletions can be identified at present by methylation analysis of the DMRs, irrespective of the karyotype. Notably, the relative frequency is grossly reminiscent of that observed in KOS14, ${ }^{21}$ but is different from that reported in other imprinting disorders. Indeed, microdeletions at the chromosome 15q11.2-q13 imprinted region are most prevalent in PWS and Angelman syndrome (AS), ${ }^{22}$ and epimutations of the H19/IGF2:IG-DMR and the KCNQ1OT1:TSS-DMR at chromosome 11p15.5 are most frequent in SRS and BeckwithWiedemann syndrome (BWS), ${ }^{23}$ respectively. In this regard, low-copy repeats flanking the imprinted region are present on chromosome 15q11.2-q13 but not on chromosomes $14 \mathrm{q} 32.2$ and $11 \mathrm{p} 15.5,{ }^{24}$ and nonmosaic UPD is apparently lethal for chromosome 11 but not for chromosomes 14 and $15 .^{21,22,25}$ This would primarily explain why microdeletions, epimutations, and UPDs are most prevalent in PWS/AS, SRS/BWS, and TS14/KOS14, respectively.

Clinical findings were grossly similar among patients with UPD(14)mat, those with epimutations, and those with microdeletions, except for significantly increased present ages in patients with microdeletions and significantly advanced maternal childbearing age in patients with UPD(14)mat. Such phenotypic similarity among UPDs, epimutations, and microdeletions has also been reported in KOS14 and PWS. $^{21,26}$ In this regard, the significantly increased present ages in patients with microdeletions are ascribed to the ascertainment bias that they were identified in adulthood by familial studies of children with KOS14, and significantly advanced maternal childbearing age in patients with UPD(14) mat is consistent with the production of disomic oocytes involved in the generation of TR/GC-mediated UPD(14)mat being a maternal age-dependent phenomenon. ${ }^{27}$

Comprehensive clinical studies were performed in all 32 patients. The results showed (i) high frequencies $(\geq 60 \%)$ of pre- and postnatal growth failure, relative macrocephaly at birth (birth OFC-SDS $\geq 1.5$ above birth length or weightSDS), prominent forehead in infancy, precocious puberty, hypotonia, small hands and feet, and feeding difficulties; (ii) intermediate frequencies (30-60\%) of relative macrocephaly at present (present OFC-SDS $\geq 1.5$ above present height or weight-SDS), high-arched palate, irregular teeth, clinodactyly, and joint hypermobility; and (iii) low frequencies $(\leq 30 \%)$ of premature delivery, triangular face, ear anomalies, recurrent otitis media, GHD, simian crease, body asymmetry, scoliosis, undermasculinized genitalia, hypercholesterolemia, and diabetes mellitus. These findings are grossly similar to those of the recent review of 51 patients with TS14. ${ }^{4}$

In addition, this study produced several notable clinical findings. First, placental hypoplasia was found with a high frequency. This is consistent with the notion that placental as well as body growth is promoted by PEGs and suppressed by $M E G s{ }^{28}$ Indeed, placental hypoplasia is frequent in SRS, ${ }^{29}$ and placentomegaly is prevalent in KOS14 and BWS cases. $^{21,23}$ The relatively large placenta in patient 21 would be due to hitherto unknown multiple (epi)genetic and environmental factors involved in placental growth (such factors may also be relevant to lack of growth failure in several patients). Second, relative macrocephaly and prominent forehead became obscure with age. This would allow for differential diagnosis between TS14 and SRS. Third, GH therapy resulted in accelerated statural growth and rapid BA progress in most patients. Thus, the effects of GH therapy on adult height remain to be clarified. Fourth, precocious puberty was confirmed to be gonadotropin-dependent, and was well treated with $\mathrm{GnRH}$ analog therapy. This provides a therapeutic option for precocious puberty in TS14. Fifth, BMIs increased along the reference curves in most patients. Thus, while the previous review indicated elevation of BMIs with age, ${ }^{4}$ the BMI data should be evaluated by sex-, age-, and ethnicity-matched BMI reference data. Finally, the prevalence of ID was apparently lower in this study than in the previous review, while delayed gross motor development ascribed to hypotonia was similarly observed in both this study and the review. In this regard, IQ/DQ remained $>70-75$ in most patients in this study and in the review, ${ }^{4}$ and low IQ/DQ was primarily observed in patients with specific backgrounds in this study. Furthermore, although speech delay was prevalent in the previous review, ${ }^{4}$ it is known that hypotonia could 
Table 2 Clinical indications for the genetic diagnosis of Temple syndrome (TS14)

General
TS14 is usually considered in patients with pre- and postnatal growth failure (and placental hypoplasia) plus the following findings.
Infantile period
- Class 1: Consider TS14 with a high priority. Coexistence of PWS-like marked hypotonia and SRS-like relative macrocephaly, prominent forehead, and
feeding difficulty.
- Class 2: Consider TS14 when the genetic causes of PWS or SRS have been excluded. PWS-like marked hypotonia only. SRS-like relative macrocephaly,
prominent forehead, and feeding difficulty only.
- Class 3: Consider TS14 as a possible underlying cause. Pre- and postnatal growth failure (and placental hypoplasia) only.
Pubertal period
- Class 4: Consider TS14 with a high priority. Precocious puberty (plus history of PWS-like and/or SRS-like phenotype in infancy).
Any age
- Class 5: Consider TS14 with a high priority.
Familial history of a patient with Kagami-Ogata syndrome.

PWS, Prader-Willi syndrome; SRS, Silver-Russell syndrome.

result in compromised quality and rate of speech because of the impaired ability to move oral structures. ${ }^{30,31}$ Indeed, although speech delay was suspected in infancy to early childhood when marked hypotonia was present, it appeared to have improved with age in this study. Thus, speech delay in infancy to early childhood might be due to marked hypotonia rather than to ID.

Clinical diagnosis was reassessed in patients with TS14 on the basis of the comprehensive clinical findings. The results suggest clinical indications for genetic testing for TS14 (Table 2). In particular, genetic testing for TS14 should be considered as a high priority for patients with pre- and postnatal growth failure (and placental hypoplasia) plus (i) both the PWS-like phenotype (e.g., marked hypotonia) and SRS-like phenotype (e.g., relative macrocephaly and feeding difficulties) in infancy, (ii) gonadotropin-dependent precocious puberty, and (iii) familial history of KOS14 caused by maternal microdeletion at the chromosome $14 \mathrm{q} 32.2$ imprinted region.

TS14 is associated with a wide phenotypic spectrum, especially in infancy. This would imply that TS14 is associated with PWS-like and SRS-like phenotypes with variable expressivity and incomplete penetrance in infancy, depending on other (epi)genetic and environmental factors. In this context, recent studies have indicated the presence of a complex molecular network among imprinted regions, including the regulatory function of IPW on 15q11.2-q13 for the expression patterns of the 14q32.2 imprinted locus. ${ }^{32}$ Thus, individual variability in such a network might underlie the (epi)genetic factors. By contrast, variable hypomethylation levels of the MEG3/DLK1:IG-DMR and the MEG3:TSS-DMR, and the presence or absence of MLIDs, would be irrelevant to phenotypic variability in patients with epimutations. While there might be abnormally methylated $\operatorname{DMR}(\mathrm{s})$ hidden in unexamined critical tissues, patient 25 with epimutation showed an almost full TS14 phenotype despite relatively mild hypomethylations of the MEG3:TSS-DMR, and no MLID was detected in patients with epimutations.
It would be worth considering the major factor(s) for the development of TS14. In this context, genotype-phenotype analysis in 16 patients with microdeletions involving the $14 \mathrm{q} 32.2$ imprinted region implies that most of the TS14 phenotypes including growth failure, PWS/SRS-like features in infancy, and precocious puberty are caused primarily by loss of DLK1 expression, whereas ID results primarily from haploinsufficiency of the nonimprinted gene(s) (YY1 has been regarded as a strong candidate for ID) ${ }^{33}$ (Figure 3). In support of this notion, DLK1 is involved in the determination of menarchial age $e^{34}$ and Dlk1 knockout mice show several TS14-like features, including pre- and postnatal growth retardation, muscular hypoplasia, and obesity. ${ }^{35,36}$ In addition, because $D l k 1$ functions as a negative regulator for adipogenesis, ${ }^{35,37}$ loss of DLK1 expression would be relevant to metabolic abnormalities observed in a few T14 patients. While case 10 in Figure 3 has SS in the presence of DLK1, this could be a coincidental feature or result from impaired DLK1 expression caused by a microdeletion involving the MEG3/ DLK1:IG-DMR. ${ }^{12}$ Loss of RTL1 expression may also have a certain role, because Rtl1 knockout mice exhibit placental hypoplasia and growth retardation. ${ }^{38}$ However, since case 13 in Figure 3 with a microdeletion involving RTL1 but not the DMRs manifests an apparently normal phenotype, further studies are required to clarify the relevance of RTL1 relating to the TS14 phenotype. By contrast, phenotypic similarity among UPD(14)mat, epimutations, and microdeletions would argue against a major role of doubled MEGs expression in phenotypic development (Supplementary Figure S1D).

Several points should be made with regard to the current study. First, TS14 may be present in patients who are born SGA and show postnatal catch-up growth or in those who are born at normal birth size show postnatal growth failure. Second, TS 14 may also be caused by microdeletions involving DLK1 but not the DMRs, or by intragenic mutations of DLK1. Third, unmasking of maternal recessive mutations in UPD(14)mat or coincidental genetic abnormalities might be hidden in patients with exceptional phenotypes 


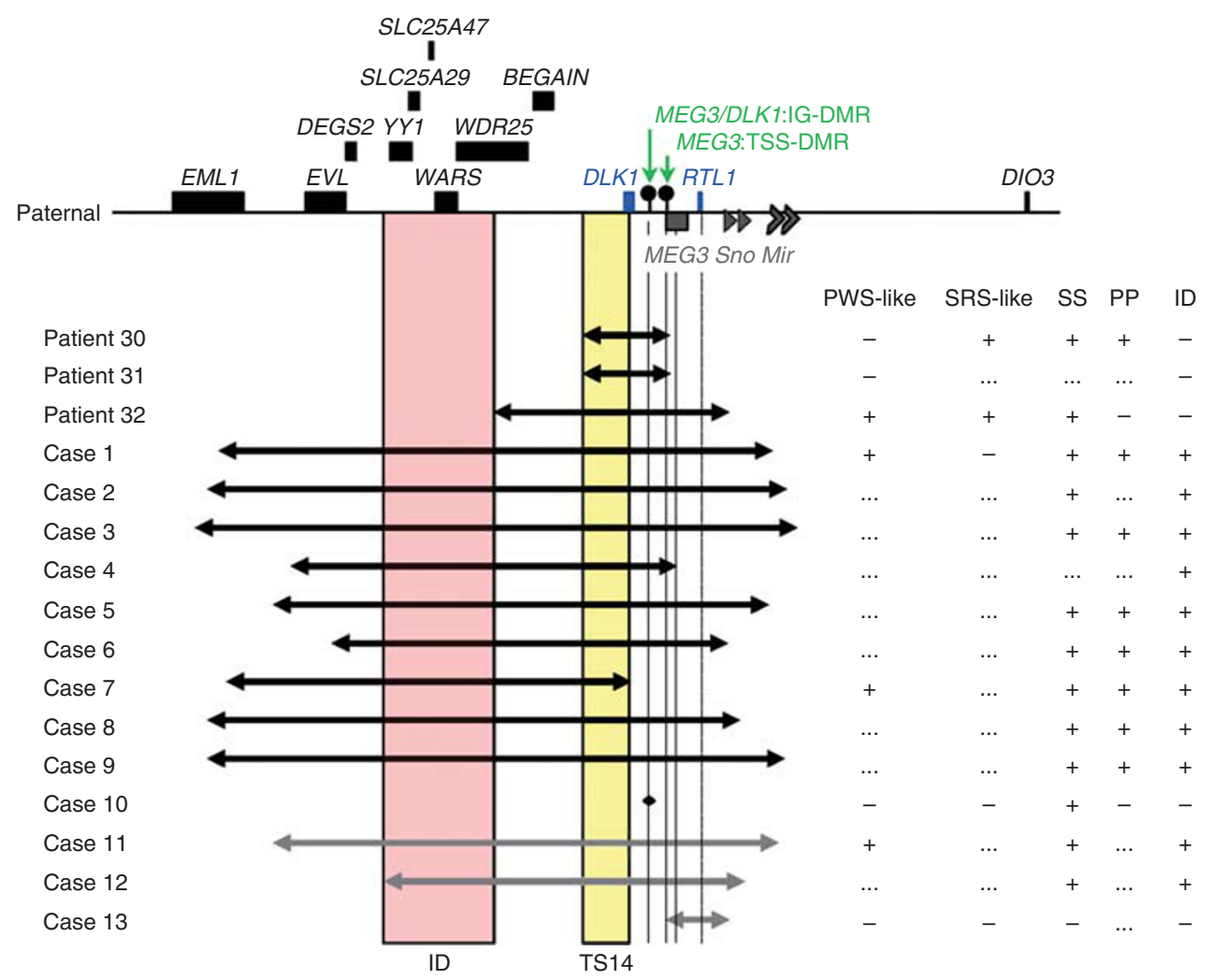

Figure 3 Genotype-phenotype correlations in 16 patients with microdeletions involving the chromosome $14 q 32.2$ imprinted region. The microdeletions were confirmed to be present on the paternally inherited chromosome 14 in patient 30-case 10 (indicated with black left-right double arrows), and are predicted to reside on the paternally inherited chromosome 14 in cases 11-13 on the basis of the phenotype (indicated with gray leftright double arrows). DLK1 and RTL1 shown in blue are PEGs, and the remaining genes shown in black are nonimprinted genes (DIO3 is unlikely to be a $P E G) .{ }^{39}$ MEGs are shown in gray because they are not expressed from the paternally transmitted chromosome 14 . The smallest overlapping region in patients with the TS14 phenotype is shaded with a yellow rectangle that contains DLK1 as the sole gene, and for patients with ID it is shaded with a pink rectangle. ID, intellectual disability; PP, precocious puberty; PWS, Prader-Willi syndrome; SRS, Silver-Russell syndrome; SS, short stature. For sources, see Supplementary Reference S2.

(e.g., neurological or emotional problems). These matters await further investigation.

In summary, we identified 32 patients with TS14 and clarified characteristic clinical findings. The results suggest that TS14 is not only a genetically diagnosed disorder but also a clinically recognizable entity with a constellation of characteristic features.

\section{SUPPLEMENTARY MATERIAL}

Supplementary material is linked to the online version of the paper at http://www.nature.com/gim

\section{ACKNOWLEDGMENTS}

We are grateful to all the patients and their parents for their cooperation. We thank Toshiro Nagai, Masayuki Yamane, Emi Okishio, Masahide Goto, Yuka Wada, Yoshio Makita, Machiko Toki, Koji Tsubouchi, Satoru Sakazume, Yasuhiro Kido, Takahiro Niizuma, Shuntaro Morikawa, Katsura Ishizu, Takanobu Inoue, Shuichi Yatsuga, and Tatsuyuki Tanaka for providing us with clinical data and materials for molecular studies, and Tamae Tanji for her support in molecular analyses. We also thank Emma
Barber for her editorial assistance with the final draft of this paper. This work was supported by grants from the Japan Society for the Promotion of Science (JSPS) (A-25253023 and 15K15096), the National Center for Child Health and Development (25-10 and 28-6), the Japan Agency for Medical Research and Development (AMED) (16ek0109030h0003 and 16ek0109141h0002), the Takeda Science Foundation, and a Japanese Society for Pediatric Endocrinology Future Development Grant.

\section{DISCLOSURE}

The authors declare no conflict of interest.

\section{REFERENCES}

1. da Rocha ST, Edwards CA, Ito M, Ogata T, Ferguson-Smith AC. Genomic imprinting at the mammalian Dlk1-Dio3 domain. Trends Genet 2008;24: 306-316.

2. Kagami M, Sekita $Y$, Nishimura $G$, et al. Deletions and epimutations affecting the human $14 q 32.2$ imprinted region in individuals with paternal and maternal upd(14)-like phenotypes. Nat Genet 2008;40: 237-242.

3. Monk D, Morales J, den Dunnen JT, et al. Recommendations for a nomenclature system for reporting methylation aberrations in imprinted domains. Epigenetics; e-pub ahead of print 2 December 2016. 
4. Ioannides Y, Lokulo-Sodipe K, Mackay DJ, Davies JH, Temple IK. Temple syndrome: improving the recognition of an underdiagnosed chromosome 14 imprinting disorder: an analysis of 51 published cases. J Med Genet 2014; 51:195-201.

5. Mitter D, Buiting $K$, von Eggeling $F$, et al. Is there a higher incidence of maternal uniparental disomy 14 [upd(14)mat]? Detection of 10 new patients by methylation-specific PCR. Am J Med Genet A 2006;140: 2039-2049.

6. Kagami M, Mizuno S, Matsubara K, et al. Epimutations of the IG-DMR and the MEG3-DMR at the $14 \mathrm{q} 32.2$ imprinted region in two patients with Silver-Russell syndrome-compatible phenotype. Eur J Hum Genet 2015:23:1062-1067.

7. Hosoki K, Kagami M, Tanaka T, et al. Maternal uniparental disomy 14 syndrome demonstrates Prader-Willi syndrome-like phenotype. J Pediatr 2009;155:900-903.

8. Kagami M, Matsubara K, Nakabayashi K, et al. Genome-wide multilocus imprinting disturbance analysis in Temple syndrome and Kagami-Ogata syndrome. Genet Med 2016;19:476-482

9. Hosoki K, Ogata T, Kagami M, Tanaka T, Saitoh S. Epimutation (hypomethylation) affecting the chromosome $14 q 32.2$ imprinted region in a girl with upd(14)mat-like phenotype. Eur J Hum Genet 2008;16: 1019-1023.

10. Tohyama J, Yamamoto $T$, Hosoki K, et al. West syndrome associated with mosaic duplication of FOXG1 in a patient with maternal uniparental disomy of chromosome 14. Am J Med Genet A. 2011;155A:2584-2588.

11. Kagami M, Kato F, Matsubara $K$, et al. Relative frequency of underlying genetic causes for the development of UPD(14)pat-like phenotype. Eur J Hum Genet 2012:20:928-932.

12. Kagami M, O'Sullivan MJ, Green AJ, et al. The IG-DMR and the MEG3DMR at human chromosome 14q32.2: hierarchical interaction and distinct functional properties as imprinting control centers. PLoS Genet 2010:6:e1000992.

13. Kagami M, Yamazawa K, Matsubara K, et al. Placentomegaly in paternal uniparental disomy for human chromosome 14. Placenta 2008;29: 760-761

14. Ogata T, Tanaka T, Kagami M. Target height and target range for Japanese children: revisited. Clin Pediatr Endocrinol 2007;16:85-87.

15. Murata M, Matsuo N, Tanaka T, et al. eds. Radiographic Atlas of Skeletal Development for the Japanese. Kanehara Press: Tokyo, Japan, 1993 (in Japanese).

16. Inada $\mathrm{H}$, Imamura $\mathrm{T}$, Nakajima $\mathrm{R}$, eds. Manual of Endocrine Examination for Children. Medical Review: Osaka, Japan, 2002 (in Japanese).

17. Yokoya S, Ogata T, Ozono K, eds. Pediatric Endocrinology, 2nd edn. Shindan-to-Chiryou-sha: Tokyo, Japan, 2016 (in Japanese).

18. Gunay-Aygun M, Schwartz S, Heeger S, O'Riordan MA, Cassidy SB. The changing purpose of Prader-Willi syndrome clinical diagnostic criteria and proposed revised criteria. Pediatrics 2001;108:E92.

19. Wakeling EL, Brioude F, Lokulo-Sodipe O, et al. Diagnosis and management of Silver-Russell syndrome: First international consensus statement. Nat Rev Endocrinol 2016;13:105-124.

20. Kotzot D. Maternal uniparental disomy 14 dissection of the phenotype with respect to rare autosomal recessively inherited traits, trisomy mosaicism, and genomic imprinting. Ann Genet 2004;47:251-260.

21. Ogata T, Kagami M. Kagami-Ogata syndrome: a clinically recognizable upd(14)pat and related disorder affecting the chromosome $14 q 32.2$ imprinted region. J Hum Genet 2016;61:87-94.

22. Gurrieri F, Accadia M. Genetic imprinting: the paradigm of Prader-Willi and Angelman syndromes. Endocr Dev 2009;14:20-28.

23. Jacob KJ, Robinson WP, Lefebvre L. Beckwith-Wiedemann and SilverRussell syndromes: opposite developmental imbalances in imprinted regulators of placental function and embryonic growth. Clin Genet 2013;84:326-334.

24. Cassidy SB, Driscoll DJ. Prader-Willi syndrome. Eur J Hum Genet 2009;17: 3-13.
25. Dutly F, Baumer A, Kayserili $H$, et al. Seven cases of WiedmannBeckwith syndrome, including the first reported case of mosaic paternal isodisomy along the whole chromosome 11. Am J Med Genet 1998;79: 347-353.

26. Matsubara K, Murakami N, Nagai T, Ogata T. Maternal age effect on the development of Prader-Willi syndrome resulting from upd(15)mat through meiosis 1 errors. J Hum Genet 2011;56:566-571.

27. Herbert M, Kalleas D, Cooney D, Lamb M, Lister L. Meiosis and maternal aging: insights from aneuploidy oocytes and trisomy births. Cold Spring Harb Perspect Biol 2015;7: 1-20.

28. Hurst LD, McVean GT. Growth effects of uniparental disomies and the conflict theory of genomic imprinting. Trends Genet 1997;13: 436-443.

29. Yamazawa K, Kagami M, Nagai T, et al. Molecular and clinical findings and their correlations in Silver-Russell syndrome: implications for a positive role of IGF2 in growth determination and differential imprinting regulation of the IGF2-H19 domain in bodies and placentas. J Mol Med 2008;86:1171-1181.

30. Martin K, Inman J, Kirschner A, et al. Characteristics of hypotonia in children: a consensus opinion of pediatric occupational and physical therapists. Pediatr Phys Ther 2005;17:275-282.

31. Lisi EC, Cohn RD. Genetic evaluation of the pediatric patient with hypotonia: perspective from a hypotonia specialty clinic and review of the literature. Dev Med Child Neurol 2011;53:586-599.

32. Patten MM, Cowley M, Oakey RJ, Feil R. Regulatory links between imprinted genes: evolutionary predictions and consequences. Proc Biol Sci 2016;283:pii: 20152760.

33. Vissers LE, de Ligt J, Gilissen C, et al. A de novo paradigm for mental retardation. Nat Genet 2010;42:1109-1112.

34. Perry JR, Day F, Elks CE, et al. Parent-of-origin-specific allelic associations among 106 genomic loci for age at menarche. Nature 2014;514:92-97.

35. Moon YS, Smas CM, Lee $K$, et al. Mice lacking paternally expressed Pref-1/Dlk1 display growth retardation and accelerated adiposity. Mol Cell Biol 2002;22:5585-5592.

36. Waddell JN, Zhang P, Wen Y, et al. Dlk1 is necessary for proper skeletal muscle development and regeneration. PLoS One 2010:5:e15055.

37. Mitterberger MC, Lechner $S$, Mattesich $M$, et al. DLK1 (PREF1) is a

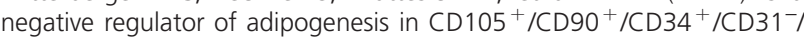
FABP4- adipose-derived stromal cells from subcutaneous abdominal fat pats of adult women. Stem Cell Res 2012;9:35-48.

38. Sekita $Y$, Wagatsuma $H$, Nakamura $K$, et al. Deletions and epimutations affecting the human $14 q 32.2$ imprinted region in individuals with paternal and maternal upd(14)-like phenotypes. Nat Genet 2008;40: 237-242.

39. Kagami M, Matsuoka K, Nagai T, et al. Paternal uniparental disomy 14 and related disorders: placental gene expression analyses and histological examinations. Epigenetics 2012;7:1142-1150.

This work is licensed under a Creative Commons Attribution-NonCommercial-NoDerivs $\quad 4.0$ International License. The images or other third party material in this article are included in the article's Creative Commons license, unless indicated otherwise in the credit line; if the material is not included under the Creative Commons license, users will need to obtain permission from the license holder to reproduce the material. To view a copy of this license, visit http://creativecommons.org/licenses/ by-nc-nd/4.0/

(c) The Author(s) 2017 